\title{
TOWARDS A CLASSIFICATION OF TEXT TYPES: A REPERTORY GRID APPROACH
}

\author{
Andrew Dillon and Cliff McKnight
}

HUSAT Research Centre, Elms Grove, Loughborough, Leics. LE11 1RG, UK.

Running head: A typology of text types.

Published in International Journal of Man-Machine Studies

This item is not the definitive copy. Please use the following citation when referencing this material: Dillon, A. and McKnight, C. (1990) Towards a classification of text types: a repertory grid approach. International Journal of Man-Machine Studies, 33, 623-636.

\begin{abstract}
The advent of hypertext brings with it associated problems of how best to present nonlinear texts. As yet, knowledge of readers' models of texts and their uses is limited. Repertory grid analysis offers an insightful method of examining these issues and gaining an understanding of the type of texts that exist in the readers' worlds. The present study investigates six researchers' perceptions of texts in terms of their use, content and structure. Results indicate that individuals construe texts in terms of three broad attributes: why read them, what type of information they contain, and how they are read. When applied to a variety of texts these attributes facilitate a classificatory system incorporating both individual and task differences and provide guidance on how their electronic versions could be designed.
\end{abstract}

, 33, 323-336 (1990). 


\section{Introduction}

The advent of hypertext has brought with it an awareness of the possibilities of structuring information in ways heretofore impossible with paper (Conklin, 1987; McAleese, 1989). While this is generally seen as advantageous, the empirical data that has so far emerged shows that people often perform better with paper than with hypertext (see for example McKnight et al., 1989). It is unlikely that one or other medium will prove better in all situations but as yet, the criteria for distinguishing between documents that would make good or bad hypertexts have not been derived.

The need for a useful typology of texts to aid such distinctions has been identified recently in the domain of hypertext research (Brown, 1988; McKnight et al., 1988). Such a typology would presumably provide a basis for distinguishing between the uses to which different texts are put and suggest the interface style required to support their hypertext equivalents. At first glance it may appear that such a typology would be relatively easy to develop. Obvious distinctions can be drawn between fiction and nonfiction, technical and non-technical, serious and humorous, etc., which discriminate between texts in a relatively unambiguous manner. However, such discriminations are not necessarily informative in terms of how the text is used or the readers' views of the contents, aspects which should be apparent from any typology aiming to distinguish meaningfully between texts.

The categorisation of texts has received some attention from linguists and typographers (see Waller, 1987, for an excellent review). For example, de Beaugrande (1980) defines a text type as "a distinctive configuration of relational dominances obtaining between or among elements of the surface text, the textual world, stored knowledge patterns and a situation of occurence” (p. 197) and offers the following illustrations: descriptive, narrative, argumentative, literary, poetic, scientific, didactic and conversational. However, de Beaugrande freely admits that these categories are not mutually exclusive and are not distinguishable on any one dimension. Waller adds that it is not at all clear where texts such as newspapers or advertisements fit in such a typology and proposes instead analysing text types in terms of three kinds of underlying structure: 
- topic structure, the typographic effects which display information about the author's argument e.g., headings;

- artefact structure, the features determined by the physical nature of the document, e.g., page size;

- access structure, features that serve to make the document usable e.g., lists of contents.

While much of this work is interesting it is concerned less with the readers and their conceptualisation of the text than with lay-out, presentation and writing style. Interdisciplinary boundaries are not always clear though, as cognitive psychologists have taken increasing interest in the relationship between so called 'typographical' features and the reading process (e.g., Hartley, 1985) and typographers look to psychology for theoretical explanations of typographic effects. Ultimately, typographers approach the problem from the practical point of view of texts and their design, not from the perspective of better understanding the reader. Their interests may, like graphic designers lie in "getting the message across" but stop short of more fully appreciating human cognition. Thus, any typographical classification of texts is likely to differ significantly from one based purely on psychological principles.

In a more psychological vein, van Dijk and Kintsch (1983) use the term “discourse types” to describe the macrostructural regularities present in real-world texts such as crime stories or psychological research reports. According to their theory of discourse comprehension such types facilitate readers' predictions about the likely episodes or events in a text and thus support accurate macroproposition formation. In other words the reader can utilise this awareness of the text's typical form or contents to aid comprehension of the material. In their view, such types are the literary equivalent of scripts or frames and play an important role in their model of discourse comprehension. However, they stop short of providing a classification or typology themselves and it is not clear how this work can be extended to inform the design of hypertext documents. 
From a less theoretical standpoint Wright (1980) describes texts in terms of their applicative domains:

- domestic (e.g. instructions for using appliances)

- functional (e.g. work-related manuals)

- advanced literacy (e.g. magazines or novels)

She uses these categories to emphasise the range of texts that exist and to highlight the fact that reading research must become aware of this tremendous diversity. This is an important point and the impetus behind the present work. Research into the presentation and reading of one text may have little or no relevance to, and may even require separate theoretical and methodological standpoints from, other texts. It is clear from the literature cited that researchers from a range of disciplines see the concept of text types as valid. The issue then is to distinguish meaningfully between texts in terms suitable for present purposes.

This study marks a first attempt at developing a classification according to readerperceived differences. The aim is to identify how readers describe their uses for, and of, different texts and the extent to which different readers perceive various texts in similar ways. In so doing it is hoped that any emerging classification of texts will provide clues as to how electronic documents can best be designed to suit readers, an intention not attributable to any of the aforementioned categorisations.

\section{Distinguishing texts: the repertory grid approach.}

Repertory grid analysis was chosen as the most suitable technique for eliciting meaningful data about how texts are viewed by readers. Developed by George Kelly (1955) as a way of identifying how individuals construe elements of their social world, Personal Construct Theory assumes that humans are basically "scientists" who mentally "represent" the world and formulate and test hypotheses about the nature of reality. There is no need to develop a detailed account of his theory here as it has been adequately presented elsewhere (e.g. Kelly, 1955; Bannister and Fransella, 1971).

The repertory grid technique has been used for a variety of clinical and non-clinical applications (e.g. studying neurotics: Ryle, 1976; magistrates’ decision making: 
McKnight, 1981; categorisation analysis: Coltheart and Evans,1982; job analysis: Hassard, 1988) and has been applied to the domain of Human Computer Interaction, particularly with respect to elicitation of knowledge in the development of expert systems (Shaw and Gaines, 1987). The technique is no longer inextricably tied to Kelly's theory of personal constructs and its use as an analytic tool does not require acceptance of the model of man which Kelly proposed (Slater 1976). However the terms Kelly used have become standard. Therefore we may describe the technique as consisting of elements (a set of "observations" from a universe of discourse), which are rated according to certain criteria termed constructs. Either or both the elements and/or the constructs may be elicited from the subject or provided by the experimenter depending on the purpose of the investigation. Traditionally both are elicited from the subject, however when a number of individuals are to be compared, it is best to provide the elements and let the subjects express their own constructs. Regardless of the method, the basic output is a grid in the form of $n$ rows and $m$ columns, which record a subject's ratings, usually on a five or seven point scale, of $m$ elements in terms of $n$ constructs.

The typical elicitation procedure involves presenting a subject with a subset of elements and asking her to generate a construct which would meaningfully (for her) facilitate comparison and discrimination between these elements. The aim is to elicit a bi-polar dimension which the subject utilises to comprehend the elements. A common example in the literature involves asking a subject to generate a construct about several people she knows. The subject might respond by generating a construct such as "like — dislike". Then all the elements (people) are rated according to this construct on a five-point scale where 1 might signify "strong like" and 5 might signify "strong dislike" etc.. As constructs are elicited and all elements subsequently rated on these, a picture of the subjects views and interpretations of a part of her world emerges.

The advantages of the technique lie in the fact that while it focuses on the individual's subjective assessment of the world, it does so in a way that (given certain scaling assumptions) readily lends itself to statistical analysis. Where subjects generate their own constructs there is unlikely to be confusion with test concepts or the terminology employed. Researchers employing this technique can expect to obtain as rich a variety of data as may be obtained from in-depth interviews but in a form that can be more easily numerically treated. In the words of Slater (1976): 
"repertory grid technique appears to offer the flexibility and individual focus characteristic of projective techniques, while also retaining the precision and quantifiability of standardised tests.”

\section{Method}

\section{Subjects:}

6 subjects ( 5 male, 1 female) had grids elicited. All were human factors professionals except for one, a mature student studying Ergonomics and working during the summer vacation at HUSAT.

Stimulus materials:

Elements were identical for all subjects and were selected on the basis of likely usage by the sample, as judged by the experimenter and several colleagues on the basis of discussions and a brief survey of similar professionals. They consisted of 9 texts:

— a newspaper (The Independent)

— a manual (Macwrite Users Guide)

— a text book (Designing the User Interface: Ben Shneiderman)

— a novel (Steppenwolf: Herman Hesse)

— a journal (Behaviour and Information Technology)

— a catalogue (Argos Catalogue Spring 1988)

— a conference proceedings (CHI '88)

— a magazine ( $M$ Magazine: The Observer Colour Supplement)

— a report (HUSAT Memo) 


\section{Procedure}

Grids were elicited individually in a quiet office. Subjects were given a brief introduction to the aims of the study and the nature of the repertory grid technique. Nine elements were presented to them to examine and with which to confirm their familiarity. Constructs were elicited using the minimal context form (Bannister and Mair, 1968) which involves presenting subjects with three elements, known as the triad, and asking them to think of a way in which two of these are similar and thereby different from the third. The triads were presented according to a predefined sequence arranged so that no pairings of elements were repeated. Subjects were expected to produce one construct per triad, however in practice several subjects produced more then one or were unable to produce any from a given triad. In the case of more than one being elicited these were noted by the experimenter and used in turn. Failing elicitation of a suitable construct from a triad the subject was given the option of receiving another triad or generating a construct spontaneously.

When a meaningful construct was generated the two poles were written on cards and placed either side of a 1-5 rating scale on the desk. Subjects then rated all the texts according to the construct, physically placing texts at some point on this scale according to their perception of its agreement with one or other pole. Once confirmation was obtained that subjects were satisfied with this arrangement the ratings were noted and the next construct elicited. The procedure halted when subjects felt that they were unable to generate further constructs or expressed a direct wish to finish.

\section{Results}

The results were analysed using the Shaw (1980) FOCUS program. This program utilises the city block metric rather than the common Euclidean metric (see Shaw, pp. 159-160, for a discussion of the rationale) and has the advantage of retaining the original construct labels of the subjects which tend to be lost with other analysis programs as emerging factors are renamed. FOCUS involves a two-way cluster analysis that systematically reorders the columns and rows of the data matrices to produce a focused grid with minimal variation between adjacent elements and constructs.

A focused grid for one subject is presented in Figure 1. The grid consists of the raw ratings made by the subjects with the element list above and the construct list below. The FOCUS program automatically reorders these to give the minimum total distance 
between contiguous element and construct rating columns. Dendrograms are constructed by joining elements and constructs at their appropriate matching levels.

******* Insert Figure 1. here *******

Figure 1: FOCUSed grid for one subject.

In Figure 1 the element dendrogram is on top and the construct dendrogram is to the right of the reordered ratings. The matching levels for both are shown on adjacent scales. The highest match between any two of the $N$ elements or $M$ constructs becomes cluster $N+1$ or $M+1$ and so forth until all elements or constructs are included at cluster $2 N-1$ in the case of $N$ elements, and $2 M-1$ in the case of $M$ constructs. Therefore, as the matching level decreases the cluster number increases.

High matches indicate that the relevant elements share identical or similar ratings on the majority of constructs or the relevant constructs discriminate identically or similarly between the majority of elements. Thus in Figure 1. it can be observed that elements 5 (journal) and 7 (proceedings) match highly and that element 4 (novel) is least similar to the others. Constructs 10 (Work - Leisure) and 1 (To do with I.T. - Not to do with I.T.) offer the highest match among the constructs elicited, while number 7 (Single author selection possible - not possible) is the lowest match. In other words the journal and the conference proceedings are seen as very similar to each other but very different from the novel by this reader, and every time a text is described as work related it also tends to be described as being about information technology. By proceeding in this manner it becomes possible to build up a detailed picture of how an individual construes texts.

In the present study all six grids were analysed (focused) together as one large grid. Shaw has developed SOCIOGRIDS as a means of comparing more than one grid elicited using identical elements but this is designed to give a measure of commonality between individuals by focusing every possible pair of grids and presenting a mode grid which represents the most highly matched constructs between subjects. In this way socionets can be drawn indicating how well the group cluster and which individuals, if any, are isolated from the remainder of the group. This was not an aspect which was directly relevant to the present investigation and it was therefore felt that FOCUS offered a more suitable means of analysis. Both methods are based on hierarchical cluster analysis and fine distinctions between them obscure the fact that the repertory grid's strength as a technique ultimately lies more in the skill of the person(s) interpreting the output than the 
particular method of analysing the data. By presenting the actual dendrograms in this paper it is possible for the reader to appreciate for themselves the interpretations of the present authors. The focused grid is too large to reproduce completely here, therefore the element and construct trees are presented separately in Figures 2 and 3.

\section{Elements}

The elements clustered into three distinct groups (see Figure 2.). These were the work related, the "news" type texts and the novel. The highest match was between the conference proceedings and the journal (90.2\%) followed by the newspaper and the magazine (85.1\%). Basically this means that any time, for example, the journal was rated as being high or low on construct $\mathrm{X}$ then the conference proceedings were rated similarly. The textbook and report both joined the first cluster at more than the 82\% matching level. This cluster eventually incorporated the software manual at the $62 \%$ level suggesting that while this manual shared some of the ratings of the other elements in that cluster it was noticeably different from them on certain constructs.

******** Insert Figure 2 here

Figure 2: Dendrogram of element clusters for all subjects.

The catalogue matched the newspaper and magazine at $69.4 \%$ which suggests that it is perceived as similar in many ways to those types of text. The novel however was the last element to be incorporated in a cluster, only linking with other elements at the $53.2 \%$ level, by which time all the other elements had formed one large cluster. This suggests that it is a unique text type among all these elements.

\section{Constructs}

Fifty-four constructs were elicited from this sample. In order to ensure that only tight clusters were identified a minimum matching level of $70 \%$ was defined as the criterion. Thus any constructs that matched below this level were ignored for the purposes of further analysis. The construct dendrogram is presented in Figure 3. Three major construct clusters emerged from this analysis which are outlined below.

\section{*****Insert Figure3 here. $* * * * * * *$}

Figure 3: Dendrogram of constructs elicited from all subjects. $(\mathrm{R})=$ reversed. 
Cluster 1: This cluster described texts which are work-related, about Human factors or Information Technology, contain technical or specific information and would be read for learning or professional purposes.

Every subject distinguished between work-related and personal reading material. All of their constructs about this distinction matched up at the 77.7\% level. The highest match was between subject 3 (work-related) and subject 5 (about Human Factors) at 94.4\%, the next highest being these with subject 2 (I.T.-related) at 88.8\%, and subject 4 (workrelated) and subject 1 (work) at $88.8 \%$. Obviously construing texts in terms of their subject matter and relevance to work is common to all subjects. The constructs "reading to learn" and "technical” matched at $88.8 \%$ and joined up with "work-related"/ "about Human Factors"/ "I.T.-related" at that level too. Also contained in this cluster were "read repeatedly" and "long-term information", matching a work-related sub-cluster at the 83.3\% level. An element that was prototypical of this construct cluster was the journal. A very poor match with this cluster was observed for the newspaper and the magazine.

Cluster 2: This contained texts that were seen as personal reading material, containing general or abstract information that would be read in a serial fashion.

The highest match in this cluster was between the constructs "abstract - applied” and “reading for pleasure - reading for need” which matched at the $94.4 \%$ level. The next highest was at the 83.3\% level between "serial" and "read from end to end". These pairs of constructs then joined at the $77.7 \%$ level. The constructs "personal benefit”, "general information" "subjective” and “wider-world interest” all matched at the 77.7\% level. These sub-clusters all joined at the $72.2 \%$ level.

These constructs suggest that certain texts are seen as more personal than work-related and contain information that is general in nature or subjectively interesting. The presence of constructs that indicate they are read in a serial fashion would indicate texts that are not intended for reference but for complete reading. A text that closely matched most of these descriptors was the novel. A very poor match with these constructs was the catalogue.

Cluster 3: This cluster described texts that were seen as having one main subject or topic, the content of which is stable and requires a high time-investment to read. Such texts are also characterised by serial reading. 
The highest match in this cluster was between the constructs "not immediately consumable” and "contains stable information” which matched at the $83.3 \%$ level. The constructs "read serially" and "one topic" matched at the $77.7 \%$ level, as did "time investment to read is high" and "single theme" . The constructs "read more than once" and "not anchored in time" matched at $77.7 \%$ and all these constructs were joined by another construct "serial" at this level too. The final construct in this cluster above the criterion level was "low occurrence of separate units in the text" which joined all of the other constructs at $72.2 \%$ level. A text that closely matched these constructs was the novel, the newspaper and magazine were typically rated as the opposite of these.

Given the high cut-off point for matching constructs that was adopted, it is not surprising that several constructs remained outside the clusters. However, in all, only 8 failed to match with any other construct at this point. These were: Read it all - Read some; Select on the basis of author - Cannot select in this way; Sequential - Non-sequential; General Specific; Explore to see if it has the answer - Know that it has the answer; Serious Trivial; Browse - Study; Text is one unit - Text is many units. Since several of these are similar to constructs that matched highly in particular clusters their omission may appear surprising. This raises two points. Firstly, the cut-off point for matching levels is decided by the experimenter and is therefore somewhat arbitrary. A matching level of $40 \%$ could have been decided upon which would then have encompassed all constructs. The point of setting a relatively high matching level such as $70 \%$ is to extract comparatively tight clusters that share much commonality. Secondly, even though the terms "sequential" and "browse" may seem very similar to other constructs such as "serial read" or "study" the manner in which they deal with the elements is the main factor in grouping constructs. Though terms might appear similar, if they do not distinguish between elements in the same way then they are unlikely to be referring to exactly the same concept.

\section{Discussion}

A large amount of data has been reduced to a more manageable level by the FOCUS package. The results demonstrate that people's manner of construing texts is complex and influenced by numerous factors. Clear distinctions between texts such as "fiction and non-fiction" have been shown to be simplistic and superficial. On a psychological level individuals are more likely to make distinctions in terms of the type of reading strategy that they employ with a text, its relevance to their work or the amount of information that a text contains. 
The construct clusters that emerged, though reasonably tight are not clear -cut. Certainly the cluster pertaining to work-related texts that contain technical and specific information and are read in order to learn or gain professional benefit is intuitively sensible given the subject sample employed. Such a cluster highlights the easily overlooked fact that much of our reading is inextricably tied up with our work and is not just a leisure activity. However the other two clusters are not so distinctive and share many similarities. Both relate to similar reading strategies (serial or sequential) with one cluster emphasising the number of topics or themes in the text and the other the fact that certain texts are read for pleasure or personal gain. These two clusters are adjacent in the focused grid and join up eventually at the $61 \%$ matching level, suggesting general similarities between them. Had a lower cut-off point been decided upon it would have been possible to interpret the construct results in terms of two main clusters rather than three.

While the terms or descriptors employed and their similarities or differences (the face validity of the output) are interesting, it is their treatment of the elements that is ultimately important. Here the results are more specific. The elements: text book, journal, conference proceedings and report all match very highly forming a particular cluster of text types. The magazine and newspaper also match very highly. These are reasonable groupings between what may broadly be termed "work" and "leisure" texts. The novel is the one text type that matches least well with all the others and once again, this appears sensible. Examining the constructs that distinguish between these texts can shed more light on the classificatory criteria employed by these subjects.

The journal and text book types are described, unsurprisingly, as work-related, about Human Factors or I.T., containing specific or technical information and are read for professional benefit or in order to extract specific information. They are likely to be read more than once and be of long-term rather than immediate use or relevance. This distinguishes them from the other two element clusters which are more likely to described as read for leisure and containing general, subjective, or non-technical information. The novel is further distinguished from the newspaper and magazine by the need to invest a lot of time and read it serially and completely. 
Elements therefore seem distinguishable on three levels:

•How they are read e.g., serially or non-serially, once or repeatedly, browsed or studied in depth, etc.

-Why they are read e.g., for professional or personal reasons, to learn or not, out of interest or out of need, etc.

-What type of information they contain e.g., technical or non-technical, about Human Factors or not, general or specific, textual or graphical, etc.

Re-examining the constructs it is possible to classify virtually all of them as referring to one of these aspects of the text. In fact the only constructs that do not seem to refer to one of these attributes are Constructs 40 (keep a written record - don't keep a written record ), 36 (keep it - don't keep it) and 19 (highly usable - not very usable) which are either very specific to the individual or bear little obvious semantic resemblance to any other construct with which they may match.

By viewing text types according to the various attributes of these three levels of use it is easy to distinguish between e.g., a novel and a journal. The former is likely to be read serially (How), for leisure (Why) and contain general or non-technical information (What) whereas the latter is more likely to be studied or read more than once (How), for professional reasons (Why) and contain technical information which includes graphics (What). This approach facilitates a classificatory system as in Figure 4.

\section{********Insert Figure 4 here ${ }^{* * * * * * * *}$}

Figure 4: A three-way classification of texts based on How, Why and What attributes.

Here, three texts are distinguished according to their positions relative to the How, Why and What axes. The descriptors study-skim, work-personal and general-specific may vary and are only intended as examples of common constructs employed by the present sample. Other samples may employ very different terms. However, these are still likely to be descriptors that pertain to the attributes How, Why and What.

Obviously there is an individualistic aspect here. The same text may be classified differently by any two readers. Thus a literary critic is likely to classify novels differently from a casual reader. Both might share similar views of how it is to be read (e.g., serially 
or in-depth) but differ in their perceptions of why it is read or what information it contains. The critic will see the novel as related to work while the casual reader is more likely to classify it as a leisure text. What it contains will differ according to the analytic skill of the reader with a critic viewing e.g., Joyce's Ulysses as an attempt to undermine contemporary English and the casual reader (if such exists) seeing it as an powerful stream of conciousness modern work. Neither is wrong; in fact both are correct. Any classification of texts based on psychological criteria must, by definition, allow for such individual differences in perception.

That readers may vary their classification of texts according to tasks (i.e. within-subject differences) was also apparent from the comments of subjects in this study. Several of them remarked that some texts could possibly be classed as work-related and personal reading depending on the situation. An obvious example of this occurs when someone reads an academic article that is both relevant to one's work and intrinsically interesting in its own right. For individuals whose professional and personal interests overlap such an occurrence was common. The present categorisation of texts allows for this by placing emphasis on the motivation for reading (the Why axis).

Allowing for both between and within-subject variance reflects the underlying psychological complexity of the categorisation. A more rigid classification based on demonstrable objective distinctions is likely to have either a very limited sphere of application outside of which it loses relevance or be as simplistic as distinctions of the form paperback - hardback or fiction - non-fiction, etc. The present classification does not therefore provide a typology of texts, but it does offer a way of distinguishing between them and analysing readers’ perceptions.

How does all this relate to the development of hypertext systems? Obviously the desire for a stable classification of texts that could have their electronic equivalents empirically decided upon and standardised remains unfulfilled. In fact, this study suggests that such a typology is probably not feasible. Theoretically, at least, one could seek to determine the complete membership of each attribute set by identifying all possible Hows, Whys and Whats and subsequently plot the range of texts that match any combination of the three. However, such a level of analysis is probably too fine-grained to be worth pursuing. Even if, for example, all possible reading styles and strategies were identified and documented (no mean feat in itself) thereby specifying the complete set of How variables, it is not at 
all clear how a similar approach could be usefully employed with the Why and What aspects.

The classification that is proposed in this paper is best seen as a simple representation of the factors influencing readers' perceptions of texts. Characterising text according to How, Why and What variables provides a useful means of understanding the manner in which a given readership is likely to respond to a text. So, for example, it could be used for understanding the similarities and differences between a telephone directory (How: skim and locate; What: specific numeric data; Why: to contact a friend) and a novel (How: serial, detailed read; What: large text; Why: leisure) and thus quickly facilitate sensible decisions about how they should be presented. Given what we know about reading from screens and HCI, the position of a novel in such a classification system would suggest that an electronic version would not be used frequently whereas an electronic telephone directory may usefully be designed to ease the process of number location or aid searching with partial information.

At a gross level, this classification may serve to guide decisions about the feasibility of developing a usable hypertext version of a text type. Where the likely readership is known this can act as a stimulus to meaningful task analysis to identify how best to design such texts. Indeed this approach has been responsible for the work reported in Dillon et al. (1989) where human factors professionals’ requirements of journals were analysed in order to arrive at informed decisions about how best to design a hypertext database of articles. In the example of the novel above, task analysis might reveal that the novel is required for teaching purposes, where sections need to be retrieved quickly and compared linguistically with other sections or writers. Obviously this would alter the How and Why attributes of the previous classification, indicating that an electronic version is now more desirable.

The classification system can be developed by further work on increasing our understanding of each of its attributes. By knowing more about why individuals access texts, how they use them in terms of reading strategies and the distinctions they make between the information type presented it should prove possible to be more specific about the question of text types. Traditionally the How question has been the domain of the psychologist, the What question the domain of the typographer and the Why question has been largely ignored. The results from this study strongly suggest that this is a mistake. Texts exist as entities in a living psychological space where motivations, interests, 
anxiety etc. are known to influence the processing strategies of the human (Eysenck, 1983). To ignore such factors is to misrepresent fundamentally the reader and user of texts and impoverish the findings of any investigation.

\section{Acknowledgement}

This work was funded by the British Library Research and Development Department as part of Project Quartet.

\section{References}

Bannister, D. and Fransella, F. (1971) Inquiring Man. Harmondsworth: Penguin.

Bannister, D. and Mair, M. (1968) The Evaluation of Personal Constructs. London: Academic Press.

de Beaugrande, R. (1980) Text, discourse and process. Norwood, NJ: Ablex.

Brown, P. (1988). Hypertext: the way forward. In J.C. Van Vliet (ed.) Proceedings of EP88. Cambridge: Cambridge University Press (in press).

Coltheart, V. and Evans, J. (1982) An investigation of semantic memory in individuals. Memory and Cognition, 9(5), 524-532.

Conklin, J. (1987) Hypertext: an introduction and survey. Computer, September, 17-41.

van Dijk, T.A. and Kintsch, W. (1983) Strategies of Discourse Comprehension.

London:Academic Press.

Dillon, A., Richardson, J. and McKnight, C. (1989) The Human Factors of journal usage and the design of electronic text. Interacting With Computers, 1(2), 183-189.

Eysenck, M. (1983) A Handbook of Cognitive Psychology. London: Lawrence Erlbaum Associates.

Hartley, J. (1985) Designing instructional text (2nd ed.) London: Kogan Page.

Hassard, J. (1987) FOCUS as a phenomenological technique for job analysis: its use in multiple paradigm research. International Journal of Man-Machine Studies, 27, 413433.

Kelly, G. (1955) The Psychology of Personal Constructs, 2 Vols. New York: Norton. McAleese, R. (ed.) (1989) Hypertext: Theory into Practice. Oxford: Intellect. 
McKnight, C. (1981) Subjectivity in sentencing. Law and Human Behavior, 5(2/3), 141147.

McKnight, C., Richardson, J. and Dillon, A. (1988) The authoring of hypertext documents. In R. McAleese (ed.) Hypertext : Theory into Practice. Oxford: Intellect.

McKnight, C., Dillon, A. and Richardson, J. (1989) A comparison of linear and hypertext formats in information retrieval. Paper presented at HYPERTEXT II, the Second Annual UK conference on Hypertext, York, July.

Ryle, A. (1976) Some clinical applications of grid technique. In P. Slater (ed.) The Measurement of Intrapersonal Space by Grid Technique. 2 vols. London:John Wiley.

Shaw, M.L.G. (1980) On Becoming a Personal Scientist. London: Academic Press.

Shaw, M.L.G. and Gaines, B. (1987) KITTEN: Knowledge initiation and transfer tools for experts and novices. International Journal of Man-Machine Studies, 27, 251-280.

Slater, P. (1976) The Measurement of Intrapersonal Space by Grid Technique. 2vols. London: John Wiley.

Waller, R (1987) The typographic contribution to language: towards a model of typographic genres and their underlying structures. Unpublished PhD Thesis, Dept. of Typography and Graphic Communication, University of Reading.

Wright, P. (1980) Textual literacy:An outline sketch of psychological research on reading and writing. In P. Kolers, M. Wrolstad and H. Bouma (eds.) Processing of Visible Language 2. London: Plenum Press. 